Check for updates

Cite this: RSC Adv., 2019, 9, 7536

Received 5th September 2018

Accepted 27th February 2019

DOI: $10.1039 / \mathrm{c} 8 \mathrm{ra07409g}$

rsc.li/rsc-advances

\section{Replacement of $n$-type layers with a non-toxic APTES interfacial layer to improve the performance of amorphous Si thin-film solar cells $\dagger$}

\author{
Hassan Hafeez, (DD $\ddagger^{a}$ Dae Keun Choi, $\hbar^{a}$ Chang Min Lee, $\ddagger^{a}$ P. Justin Jesuraj, (D) a \\ Dong Hyun Kim, ${ }^{a}$ Aeran Song, ${ }^{b}$ Kwun Bum Chung, (D) b Myungkwan Song, ${ }^{c}$ \\ Jun Fei Ma, ${ }^{c}$ Chang-Su Kim ${ }^{\star c}$ and Seung Yoon Ryu (D) *a
}

\begin{abstract}
Hydrogenated amorphous Si (a-Si:H) thin-film solar cells (TFSCs) generally contain p/n-type Si layers, which are fabricated using toxic gases. The substitution of these $\mathrm{p} / \mathrm{n}$-type layers with non-toxic materials while improving the device performance is a major challenge in the field of TFSCs. Herein, we report the fabrication of a-Si:H TFSCs with the n-type Si layer replaced with a self-assembled monolayer (3aminopropyl) triethoxysilane (APTES). The $\mathrm{X}$-ray photoelectron spectroscopy results showed that the amine groups from APTES attached with the hydroxyl groups $(-\mathrm{OH})$ on the intrinsic Si (i-Si) surface to form a positive interfacial dipole towards i-Si. This interfacial dipole facilitated the decrease in electron extraction barrier by lowering the work function of the cathode. Consequently, the TFSC with APTES showed a higher fill factor (0.61) and power conversion efficiency (7.68\%) than the reference device (without APTES). This performance enhancement of the TFSC with APTES can be attributed to its superior built-in potential and the reduction in the Schottky barrier of the cathode. In addition, the TFSCs with APTES showed lower leakage currents under dark conditions, and hence better charge separation and stability than the reference device. This indicates that APTES is a potential alternative to n-type Si layers, and hence can be used for the fabrication of non-toxic air-stable a-Si:H TFSCs with enhanced performance.
\end{abstract}

\section{Introduction}

The photovoltaic technology, which converts solar energy into electricity has been extensively studied, ${ }^{\mathbf{1 , 2}}$ leading to the development of hydrogenated amorphous $\mathrm{Si}(\mathrm{a}-\mathrm{Si}: \mathrm{H})$ thin film solar cells (TFSCs)., ${ }^{3,4}$ They are favorable due to their simple and inexpensive fabrication, possibility of deposition on flexible/bendable substrates ${ }^{5,6}$ and the highly transparent nature has been recently exploited for "building integrated photovoltaic (BIPV)" systems. ${ }^{7,8}$ However, the greatest challenge in further development of high performance a-Si:H TFSCs is without (w/o) the involvement of hazardous/toxic doping gases., ${ }^{\mathbf{5 , 6 , 9 , 1 0}}$ The fabrication of such devices should be carried out under extremely safe environments. In addition, the use of hazardous/toxic doping gases causes device instabilities because of carrier trapping and the formation

${ }^{a}$ Division of Display and Semiconductor Physics, Display Convergence, College of Science and Technology, Korea University Sejong Campus, 2511 Sejong-ro, Sejong City, 30019, Republic of Korea. E-mail: justie74@korea.ac.kr; cskim1025@kims.re.kr ${ }^{b}$ Division of Physics and Semiconductor Science, Dongguk University, Seoul 04620, Republic of Korea

${ }^{c}$ Advanced Nano-Surface Department, Korea Institute of Materials Science (KIMS), Changwon, 51508, Republic of Korea

$\dagger$ Electronic supplementary information (ESI) available. See DOI: 10.1039/c8ra07409g

\$ These authors contributed equally to this paper. of Si dangling bonds. ${ }^{5,6}$ Many alternate processes have been investigated and toxic-free layers have been utilized using various processes in the fabrication of a-Si:H TFSCs. For example, Jung et al. ${ }^{9}$ demonstrated the deposition of a $\mathrm{V}_{2} \mathrm{O}_{5-x}$ window layer using RF magnetron sputtering as an efficient replacement of $\mathrm{p}$ type silicon layer resulting in a power conversion efficiency (PCE) of about $7.04 \%$. Ryu et al. ${ }^{5}$ presented the scheme for replacement of both p- and n-type layers with $\mathrm{MoO}_{3}$ and $\mathrm{LiF}$, respectively processed with thermal deposition achieving a PCE of $\sim 7.08 \%$. Furthermore, in crystalline silicon solar cells, Battaglia et al. ${ }^{\mathbf{1 1}}$ substituted the p-type Si layer with $\mathrm{MoO}_{3}$ and achieved a PCE of $14 \%$ while, Bullock et al. ${ }^{12}$ presented a maximum efficiency of $20 \%$ by incorporating dopant-free alkali metal fluorides and metal oxides for electron and hole selective carriers, respectively. Masuda et al. ${ }^{13}$ exhibited a completely toxic-free and solutionprocessable method for the deposition of p-type, n-type and amorphous (intrinsic) i-Si layers. However, the low PCE (0.3$0.51 \%$ ) makes it unsuitable for the commercial adoption and therefore, the exploration of other alternatives is highly desirable.

Among all the alternatives, materials with amine groups $\left(-\mathrm{NH}_{2}\right)$ have drawn greater attention as they are not only dopantfree but are also capable of generating an interfacial dipole, which can effectively tune the work function $(\varphi)$ of the electrodes. ${ }^{\mathbf{6} 14}$ In this regard, Song et al. ${ }^{15}$ proposed a promising approach to fabricate air-stable inverted organic solar cells using 
a self-assembled monolayer (SAM) of 3-aminopropyltriethoxysilane (APTES). However, it is to be noted that in majority of the proposed methods, the orientation of the APTES molecule initiated with -Si binding itself on the substrate and $-\mathrm{NH}_{2}$ facing outwards close to the cathode. ${ }^{15,16}$ This resulted in the generation of a positive interfacial dipole ${ }^{17}$ from the cathode towards the active layer. Interestingly, in this study, the orientation of APTES was in the opposite direction, i.e. the $-\mathrm{NH}_{2}$ groups bonded with the hydroxyl $(-\mathrm{OH})$ groups present on the substrate through hydrogen bonding, as revealed by the X-ray photoelectron spectroscopy (XPS) and as demonstrated by Acres et al. ${ }^{18}$ In this study, we investigated the performance of the SAM interfacial layer (APTES) with inverse orientation as an alternative to n-type layers for a-Si:H TFSCs. To the best of our knowledge such an investigation has not been carried out till date. The APTES SAM generated an interfacial dipole, lowering the $\varphi$ of the cathode. This lowering of $\varphi$ improved the charge extraction from the active layer. The devices with an APTES n-type interfacial layer showed significantly higher fill factors (FFs), PCEs, and stability (low leakage current) than the TFSCs w/o APTES.

\section{Experimental}

\section{Materials and methods}

Textured fluorine-doped tin oxide (FTO, Pilkington)-coated glass (Type NSG TECTM 7 with a sheet resistance of 6-8 $\Omega$ $\mathrm{sq}^{-1}$, thickness of about $3 \mathrm{~mm}$, visible light transmittance $80-$ $82 \%$ ) was cleaned by ultrasonication and rinsing in ethanol and deionized water (DI) for $15 \mathrm{~min}$. The intrinsic Si (i-Si) layer (500 $\mathrm{nm})$ was deposited using a mixture of silane $\left(\mathrm{SiH}_{4}\right)$ and hydrogen $\left(\mathrm{H}_{2}\right)$ (50 sccm/50 sccm) with chamber's working pressure of 400 mTorr with a very high frequency (VHF, 40.7 $\mathrm{MHz}$ ) and power of $20 \mathrm{~W}$. The p-type Si layer (12 nm) was deposited under the RF power of $50 \mathrm{~W}$ using a combination of different gases including $\mathrm{SiH}_{4} / \mathrm{H}_{2}(30 \mathrm{sccm} / 150 \mathrm{sccm})$ and $\mathrm{B}_{2} \mathrm{H}_{6}$ (9 sccm). For both layers, the plasma-enhanced chemical vapor deposition (PECVD) power density was maintained at $0.074 \mathrm{~W}$ $\mathrm{cm}^{-2}$ keeping the electrode dimensions in consideration. Approximately $15.7 \mathrm{~mm}$ distance was maintained between the shower head and the upper electrode at $250{ }^{\circ} \mathrm{C}$.

In order to develop a silanol-terminated i-Si layer, we used ultra-violet ozone at $187 \mathrm{~nm}$ for $20 \mathrm{~min}$, which increased the amount of $\mathrm{O}$ and $\mathrm{H}$ components on the surface. The APTES solution was purchased ( $\geq 98 \%$ purity, Sigma Aldrich, USA) with a shelf-life of 2 years and was used w/o any further processing. A thin APTES layer $(\sim 2 \mathrm{~nm})$ was spin coated on the i-Si layer at $2000 \mathrm{rpm}$ for $40 \mathrm{~s}$ followed by drying ${ }^{18}$ for $60 \mathrm{~s}$. The APTES layer is usually unstable under intense environmental conditions such as presence of moisture, high humidity and any other chemical substances, ${ }^{19}$ therefore, to eliminate any possible adverse effects, the deposition process was conducted in a glove box with a precise control of humidity/temperature. After deposition of APTES layer, the substrate was immediately transferred to the vacuum chamber where a double layer of $\mathrm{LiF}$ $(1.5 \mathrm{~nm}) / \mathrm{Al}(100 \mathrm{~nm})$ was deposited as the cathode at a base pressure of $\sim 2 \times 10^{-6}$ torr. A shadowed mask was used by thermal evaporation to define the active area $\left(0.25 \mathrm{~cm}^{2}\right)$ of the cell. The overall process ensured that the APTES layer was not considerably exposed to any harsh environmental/chemical conditions and indicates its adequate stability.

\section{Characterizations}

The surface morphology of the deposited APTES layer was examined using a multimode 8 atomic force microscope (AFM, Bruker) equipped with a Nanoscope V controller. The AFM measurements were carried out in the tapping mode at room temperature with $384 \times 384$ pixels over a scan size of $3 \times 3 \mu \mathrm{m}$ at a scan rate of $1 \mathrm{~Hz}$. We used an Al-coated silicon probe (NCHR, Nanoworld, Germany) with a nominal spring constant of $40 \mathrm{~N} \mathrm{~m}^{-1}$ and a resonance frequency of $320 \mathrm{kHz}$. The compositional analysis was carried out using X-ray photoelectron spectroscopy (XPS, VG Microtech ESCA 2000). An Al Ka photon source was used to examine the composition of the APTES layer. The chemical bonding states of the layers were investigated in detail using their N1s and Si2p spectra normalized and de-convoluted with difference Gaussian peaks. Ultraviolet photoelectron spectroscopy (UPS) analysis was carried out at an energy resolution of $0.05 \mathrm{eV}$ and the samples were biased at $-\mathbf{1 5 . 0} \mathrm{V}$. The performance of the a-Si:H TFSCs under light and dark conditions was evaluated using a Keithley 2400 source meter with an intensity of $100 \mathrm{~mW} \mathrm{~cm}^{-2}$. The simulated illumination using a solar simulator (Peccell Technologies, PEC-L11) was carried out under AM1.5. The external quantum efficiency of the devices was measured using an incident photocurrent efficiency measurement system equipped with a $200 \mathrm{~W}$ Xe lamp and a grating monochromator. The light intensity measurements were carried out using a calibrated Si solar cell. The device efficiency data in Table 1 presents the average performance from 5 devices with a cell active area of $0.25 \mathrm{~cm}^{2}$ and the approximate yield of the devices was observed to be more than $60 \%$. The surface wettability of the APTES layer was analyzed using the smart drop standard (FEMTOBIOMED, South Korea). The contact angle (CA) system was integrated with single non-approximated algorithm numerical calculations of Bash forth-admans (equation) and was free from disturbance. An ultra high-resolution field emission scanning electron microscope (UHR FE-SEM, S-5500, Hitachi, at Korea Basic Science Institute, Jeonju Center) was used at an accelerating voltage of $7 \mathrm{kV}$ for the analysis of the surface morphology of the layers.

\section{Results and discussion}

Fig. 1(a) the schematic of the a-Si:H TFSCs device structure using the conventional layout (textured fluorine-doped tin oxide (FTO, Pilkington)/p-type Si/intrinsic Si (i-Si)/APTES/lithium fluoride (LiF)/Al). Due to the requirement of toxic/hazardous doping gases in the processing of n-type layer deposition, APTES was utilized as the replacement. This SAM layer consists of a specific molecular arrangement owing to the presence of $-\mathrm{NH}_{2}$, silane, and carboxyl groups $(-\mathrm{C}=\mathrm{O}) .{ }^{15,20,21}$ The positive and negative charge distributions on $-\mathrm{NH}_{2}$ and $-\mathrm{O}$, respectively created a dipole moment, which depended on the electron 
Table 1 APTES TFSCs device performance (including standard deviation) comparison with various device structures including bare i-Si, P-I-N and PFN

\begin{tabular}{|c|c|c|c|c|c|c|}
\hline Device structure & $J_{\mathrm{sc}}\left(\mathrm{mA} \mathrm{cm}{ }^{-2}\right)$ & $V_{\text {oc }}(\mathrm{V})$ & FF (fill factor) & PCE (\%) & $R_{\mathrm{s}}\left(\Omega \mathrm{cm}^{2}\right)$ & $R_{\mathrm{SH}}\left(\Omega \mathrm{cm}^{2}\right)$ \\
\hline FTO/P-I Si/Al & $13.82 \pm 0.12$ & $0.63 \pm 0.01$ & $0.48 \pm 0.01$ & $4.30 \pm 0.06$ & 18.20 & $3.2 \times 10^{3}$ \\
\hline FTO/P-I Si/APTES/Al & $14.42 \pm 0.10$ & $0.75 \pm 0.01$ & $0.50 \pm 0.01$ & $5.52 \pm 0.05$ & 27.60 & $7.35 \times 10^{3}$ \\
\hline FTO/PIN-Si/Al ${ }^{a, 10}$ & 15.15 & 0.82 & 0.61 & 7.60 & 6.4 & $6.1 \times 10^{3}$ \\
\hline $\mathrm{FTO} / \mathrm{PI}-\mathrm{Si} / \mathrm{PFN} / \mathrm{Al}^{a, 6}$ & 15.04 & 0.80 & 0.59 & 7.17 & 19.0 & $1.1 \times 10^{5}$ \\
\hline FTO/P-I Si/APTES/LiF/Al & $15.51 \pm 0.08$ & $0.80 \pm 0.01$ & $0.61 \pm 0.01$ & $7.68 \pm 0.03$ & 11.70 & $9.52 \times 10^{3}$ \\
\hline
\end{tabular}

${ }^{a}$ The data has been reported before, using the same fabrication tool and conditions as mentioned in this work and was added in the table for comparison purpose.

withdrawing and donating natures of the atoms placed transversely in the molecule (Fig. 1(b)). ${ }^{\mathbf{1 6}}$ It is well-known that in APTES-related processes, the silane present in APTES attached itself to the substrate through hydrogen bonding, while the $-\mathrm{NH}_{2}$ groups pointing outwards contribute to the generation of an interfacial dipole with a dipole direction opposite to the direction of electron extraction. ${ }^{\mathbf{1 5 , 1 6}}$ However, in this study, the molecular orientation of the APTES deposited on i-Si was reversed (Fig. 1(b)), where $-\mathrm{NH}_{2}$ attached to the $-\mathrm{OH}$ from i-Si through hydrogen bonding while silane and $-\mathrm{C}=\mathrm{O}$ (EtO) acted as the terminal groups near the LiF/Al cathode. Consequently, an interfacial dipole with the dipole direction opposite to the direction of electron extraction was obtained thus reducing the electron extraction barrier $(\delta)$. The presence of these functional groups and orientation was analyzed in detail using XPS (Fig. 2) with full survey shown in Fig. S1. $\dagger$ Fig. 2(a) shows the Si2p XPS spectra of the bare and APTES-treated i-Si layers. The APTES-treated i-Si layer showed $\mathrm{Si}-\mathrm{O}$ bonds $(100 \%$ contribution) at $\sim 103 \mathrm{eV}^{22}$ The bare i-Si sample showed an additional Si-O peak with $\sim 22 \%$ contribution at $\sim 102 \mathrm{eV}$

(a)
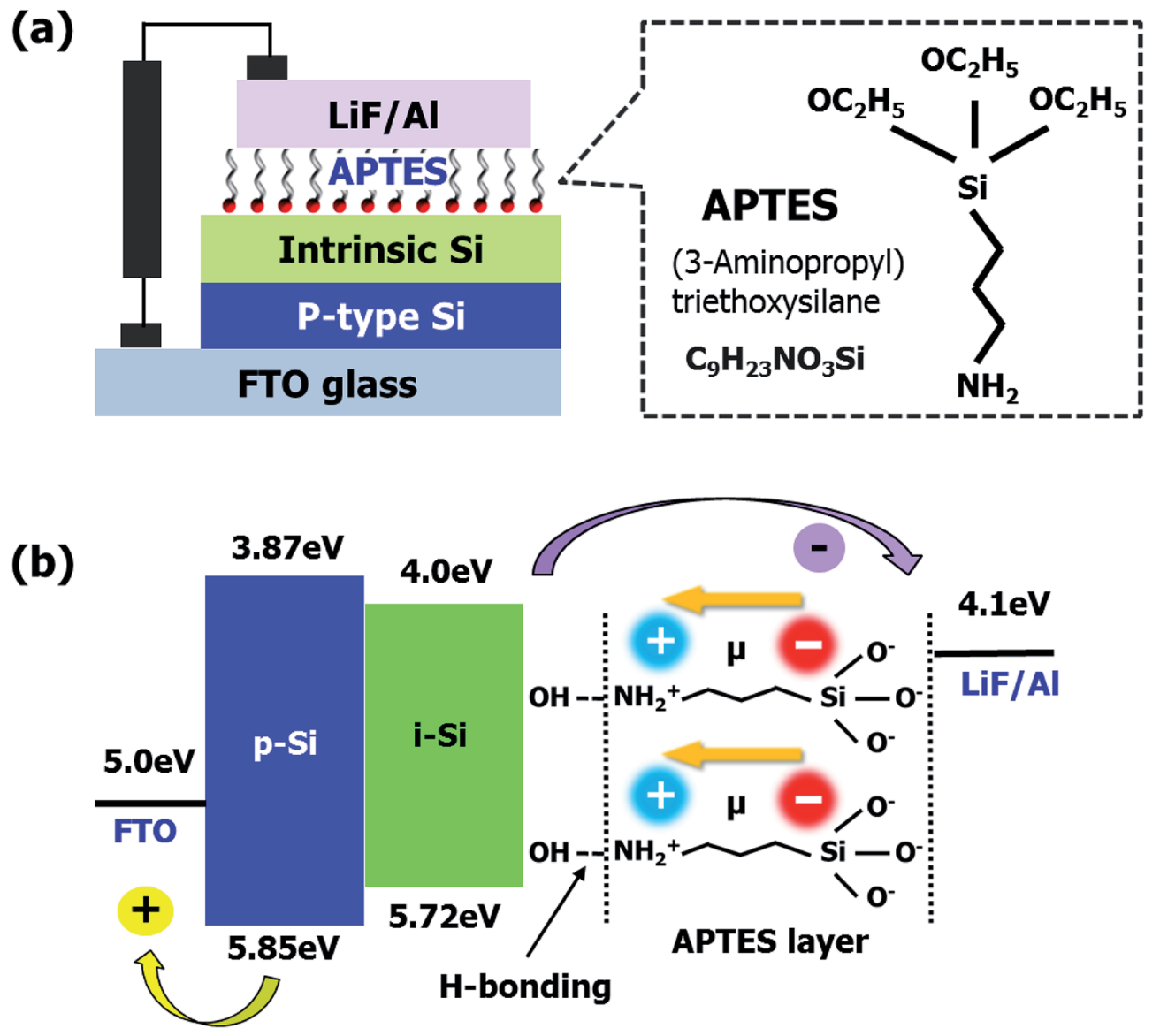

Fig. 1 (a) Schematic of the a-Si:H TFSCs with APTES as the $n$-type layer. Chemical structure of APTES demonstrating the presence of - NH ${ }_{2}$, $-\mathrm{Si}$, and $-\mathrm{C}=\mathrm{O}$ functional groups with an inverse orientation and dipole direction towards the $\mathrm{i}$-Si layer. (b) Band-gap alignment of the layers used in the devices with APTES as an interfacial layer between the i-Si layer and the cathode demonstrating the arrangement of APTES molecules on the i-Si layer and the direction (yellow arrow) of the interfacial dipole. 
(Fig. 2(d)) along with the bulk Si peak at $\sim 99 \mathrm{eV} \cdot{ }^{23}$ This could be attributed to the oxidation/hydroxylation of bare i-Si, leading to the availability of $-\mathrm{OH}$ groups on the layer surface. The N1s profile $^{\mathbf{1 5}}$ of the APTES-treated i-Si layer showed the presence of free $-\mathrm{NH}_{2}$ groups $(\sim 400 \mathrm{eV})$ from APTES (Fig. 2(a)). The deconvolution process (Fig. 2(c)) revealed the presence of an additional $-\mathrm{NH}_{2}$ peak at $\sim 401 \mathrm{eV}$ (with $20 \%$ contribution) corresponding to the hydrogen-bonded $-\mathrm{NH}_{2}\left(\mathrm{NH}_{3}\right) .{ }^{18}$ This analysis provided a concrete evidence for the inverse molecular orientation of APTES on i-Si, as mentioned above and reported previously. ${ }^{18}$ Regarding the nature of the APTES bonding with the i-Si surface, Kim et al. ${ }^{24}$ reported that the APTES films could be formed in both covalent and non-covalent (physical bond) manners, however if the process is carried out using aqueous solutions, the greater probability favors the formation of hydrogen bonds or electrostatic interactions (van der Waals). In our work, the APTES layers were deposited using spin coating of the APTES solution onto the i-Si substrate, therefore we could speculate the establishment of hydrogen bonds between APTES
$\left(-\mathrm{NH}_{2}\right)$ and $-\mathrm{OH}$ from the i-Si substrate. The deposition process for device fabrication was carried out in a glove box, however for UPS/XPS analysis, the samples were taken out of the chamber and transferred. As APTES is a hydrolytically unstable material, ${ }^{25}$ an exposure to air/moisture might have disengaged the weak hydrogen bonds or even possibly reoriented the molecules due to the change in molecular environment. ${ }^{18}$ Therefore, the observed contribution (20\%) only provides an indication about the possible orientation of the molecules on the surface. However, if we may perform XPS/UPS analysis within the controlled environmental conditions (in situ measurement), it might be possible to observe an appropriate percentage of the $\mathrm{NH}_{2}-\mathrm{H}$ bonding. Overall, this orientation of APTES on i-Si was found to be coherent with the attachment possibilities of APTES on Si substrates demonstrated by Acres et al. ${ }^{18}$ (Fig. S2 $\dagger$ ) and is also in accordance with the decrease in work function observed due to the interfacial dipole (towards i-Si). The surface morphologies of the bare and APTES-treated i-Si layers were examined using atomic force microscopy (AFM, Fig. S3†) and
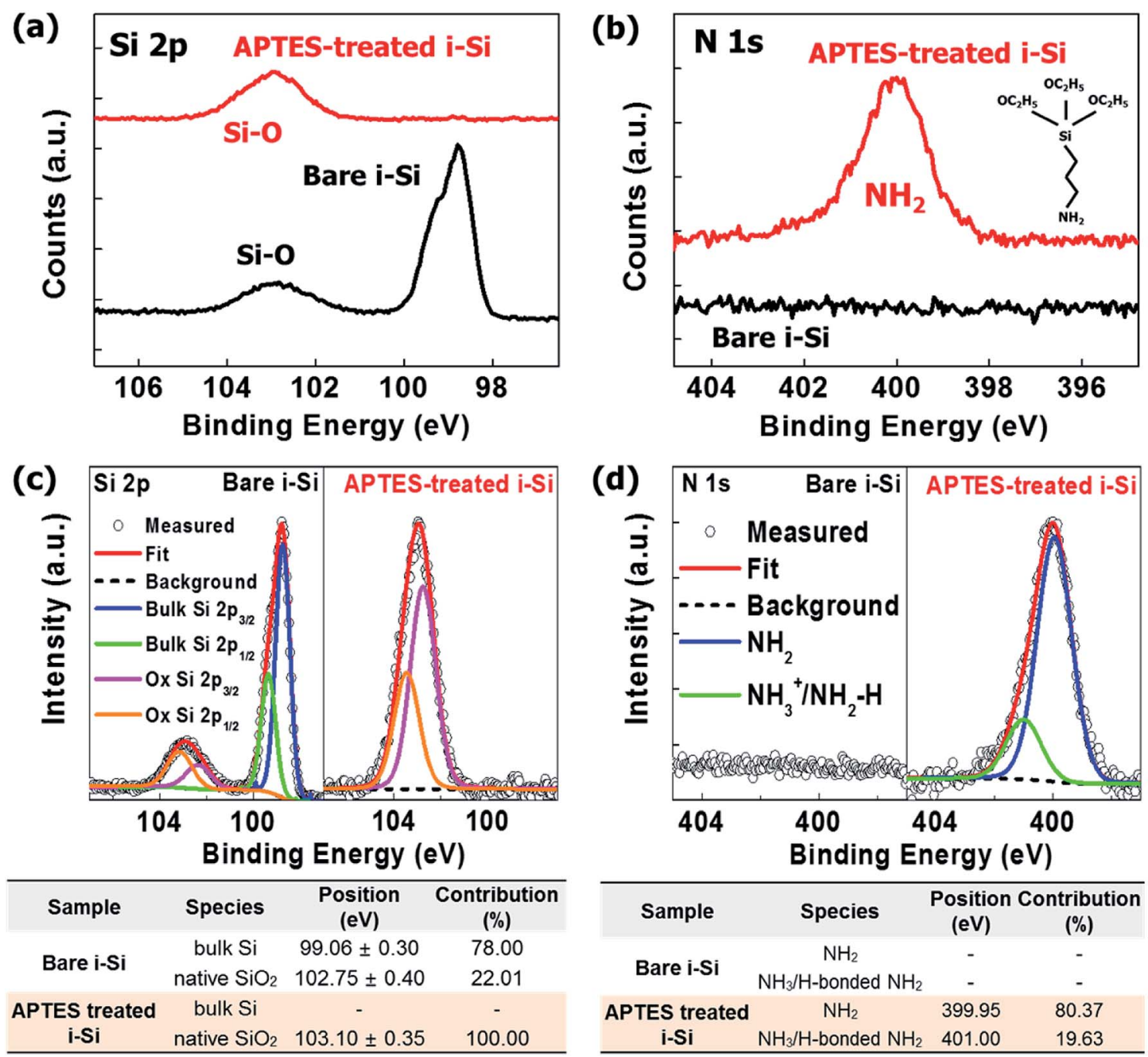

Fig. 2 High resolution (a) Si2p and (b) N1s XPS profiles of the bare i-Si and APTES-treated i-Si layers to analyze the composition of the layer and nature of bonds. (c) Si2p and (d) N1s spectra were carefully normalized and de-convoluted with difference Gaussian peaks and the table summarizes the contribution of the analyzed functional groups and bonds. 
were further analyzed by the ultra-high-resolution field emission scanning electron microscopy (UHR FE-SEM, Fig. S4 $\dagger$ ). Because of the underlying FTO with a surface roughness of $30.81 \pm 0.3 \mathrm{~nm},{ }^{26}$ the bare i-Si layer samples demonstrated a matching surface roughness (root mean square (RMS) $=$ $\sim 30.21 \pm 0.1 \mathrm{~nm})$ and hence a higher contact angle $\left(\mathrm{CA}=\sim 63^{\circ}\right)$ of water droplet with the surface was observed (Fig. S4 $\dagger$ ). The RMS value for the APTES-based i-Si layer $(29.69 \pm 0.1 \mathrm{~nm})$ was also found to be almost similar indicating a uniform deposition of the layer. However, a significant decrease in the CA (to $\sim 29^{\circ}$ ) of the APTES-treated i-Si layer was because of the presence of an interfacial dipole (chemical aspect, Fig. S4†) generated by the APTES layer.

Fig. 3(a and b) shows the changes in the Fermi level (Fermi edge) of the i-Si layer after the deposition of the APTES layer. The i-Si layer had a $\varphi$ of $\sim 1.72 \mathrm{eV}$, and the deposition of APTES resulted in the generation of interface dipoles in the i-Si/ cathode interface, which in turn reduced the electron interface barrier significantly. This improved the vacuum level between the i-Si layer and the cathode, which caused a reduction of $\sim 0.5 \mathrm{eV}$ in the electron barrier. Even though, the $\varphi$ changes were observed for the i-Si layer and not for the LiF/Al cathode, and the reduction was oddly high (1.2 eV) (Fig. 3(c and d)), which could be due to the un-wanted oxidation of the iSi layer owing to delayed ultraviolet photoelectron spectroscopy (UPS) measurement. However, APTES deposition reduced the $\varphi$ of the cathode by $\sim 0.5 \mathrm{eV} .{ }^{15}$ The analysis suggests that the deposition of APTES resulted in the reduction of the Schottky barrier at the interface between the i-Si layer and the cathode.
This improved the built-in potential $\left(V_{\mathrm{bi}}\right)$ and band-gap alignment for efficient electron extraction, while suppressing the charge recombination. ${ }^{6}$ This enhancement could be observed from the current density-voltage $(J-V)$ characteristics of the aSi:H TFSCs analyzed under light (Fig. 4(a)) and dark conditions (Fig. 4(b)). The device performance parameters such as the short-circuit current $\left(J_{\mathrm{sc}}\right)$, open-circuit voltage $\left(V_{\mathrm{oc}}\right), \mathrm{FF}$, and PCE are summarized in Table 1 . The FTO/PIN-Si/Al ${ }^{\mathbf{1 0}}$ device performance incorporated for comparison in the table were realized using the same fabrication tool and conditions as mentioned in this work and therefore can be suitably utilized for evaluation purposes. The comparison of devices w/o and with APTES using Al cathode demonstrated the worst $J_{\mathrm{sc}}\left(13.82 \mathrm{~mA} \mathrm{~cm}^{-2}\right)$ and $V_{\mathrm{oc}}$ $(0.63 \mathrm{~V})$, which was improved to $14.42 \mathrm{~mA} \mathrm{~cm}^{-2}$ and $0.75 \mathrm{~V}$, respectively with the insertion of APTES. In general, the overall lower $V_{\text {oc }}$ observed for all devices could be further enhanced by proper optimization of the process conditions for deposition of i-Si.

Also, the shunt resistances $\left(R_{\mathrm{SH}}\right)$ of the devices with and w/o APTES were found to be $7.35 \times 10^{3}$ and $3.2 \times 10^{3} \Omega \mathrm{cm}^{2}$, respectively. This indicates that APTES reduced the $\varphi$ of the cathode, and hence enhanced the electron extraction and $V_{\mathrm{bi}}$ by the generation of an interfacial dipole. However, a discrepancy in terms of series resistance $\left(R_{\mathrm{S}}\right)$ was observed, where despite of providing a higher $V_{\text {oc }}$, the APTES-treated devices demonstrated a high $R_{\mathrm{S}}$ of $\sim 27.60 \Omega \mathrm{cm}^{2}$ as compared to the device w/o APTES $\left(18.20 \Omega \mathrm{cm}^{2}\right.$ ) (Table 1$)$. This can be attributed to the reverse polarization of the APTES molecules near the surface of the Al cathode, which occurred because of the direct contact of the
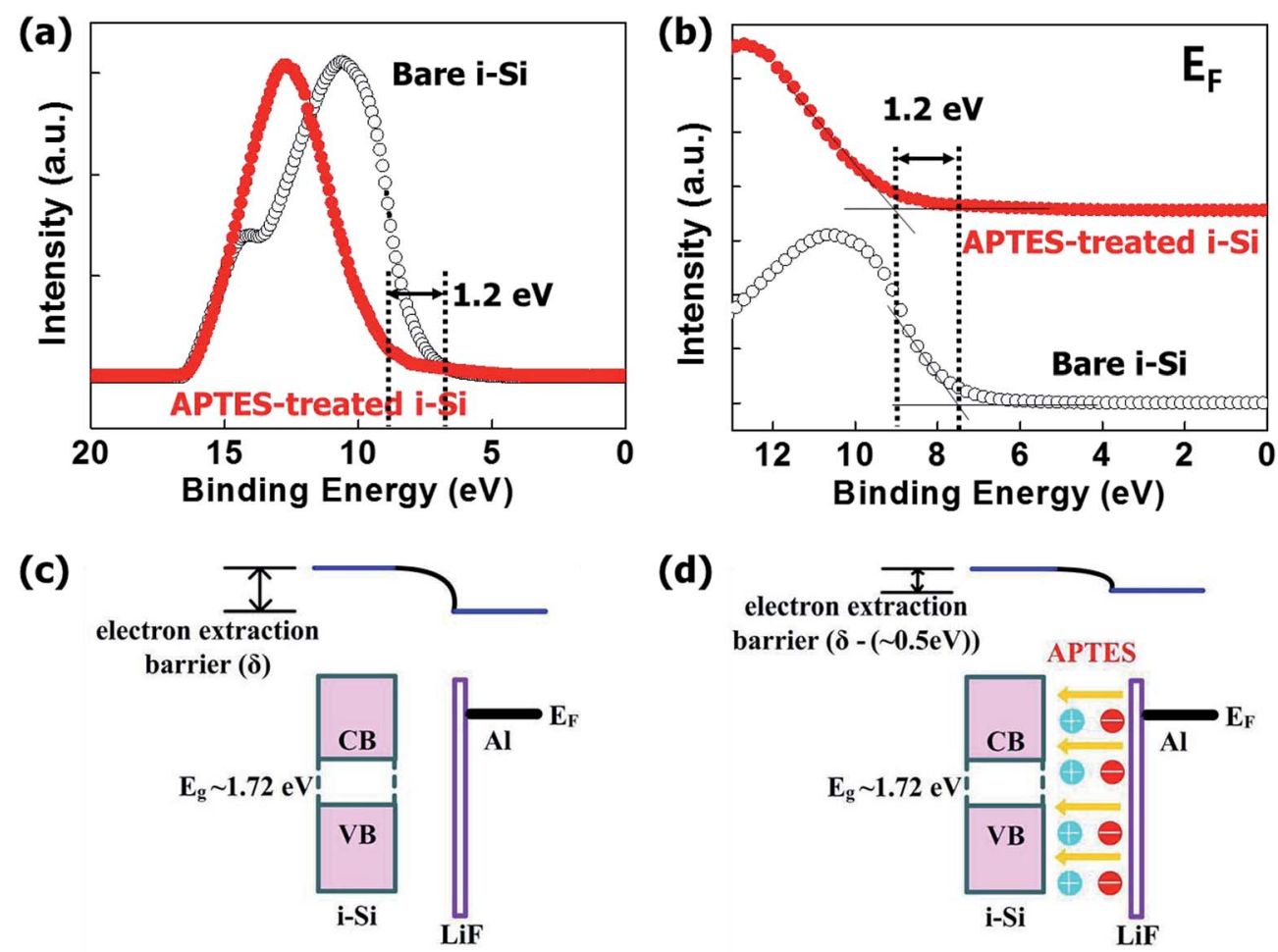

Fig. 3 (a) Normalized UPS spectra showing the Fermi levels of the bare i-Si and APTES-treated i-Si layers (b) enlarged (smaller scale) spectra to demonstrate the difference between the Fermi edges (c) $\delta$ at the interface w/o APTES, (d) reduced $\delta$ upon the addition of APTES layer. 
(a)

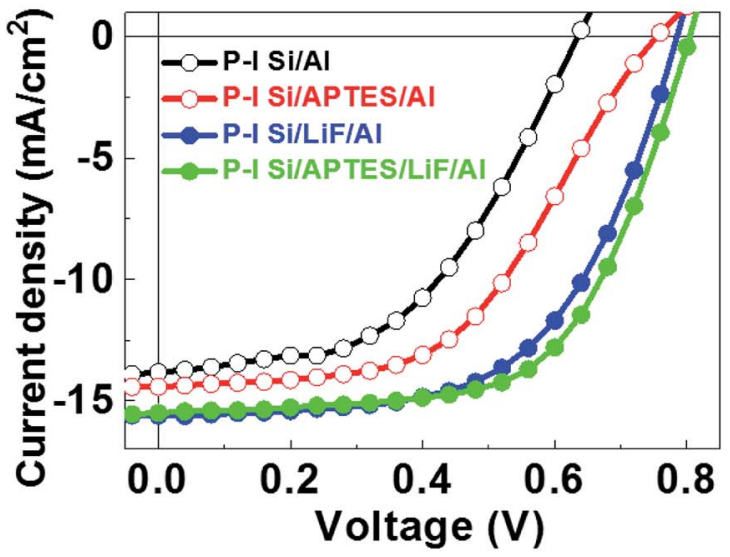

(b)

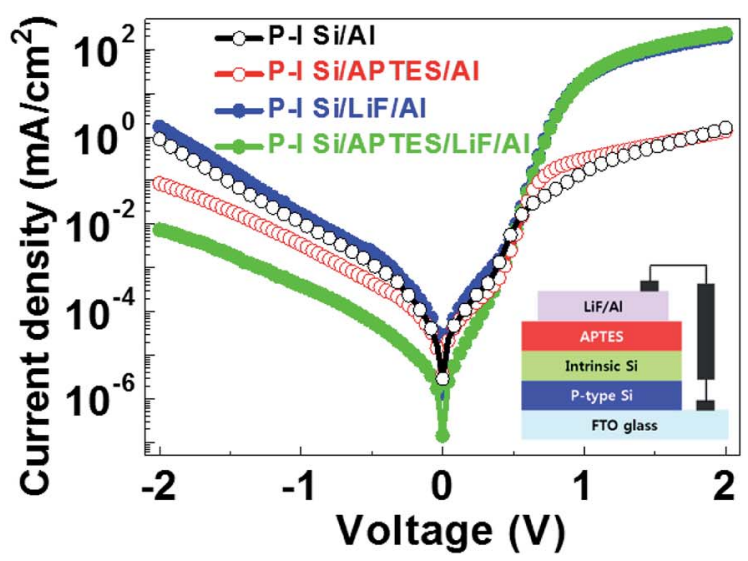

Fig. 4 Current density-voltage ( $J-V$ ) curves of the devices $w / o$ and with APTES layer, also the comparison with the presence/absence of $\mathrm{LiF}$ at the cathode (a) under illumination (b) under dark conditions.

SAM layer with the metal surface (Fig. $\mathrm{S} 5 \dagger) .{ }^{17}$ The polarization of APTES molecules reversed the dipole direction at the APTES/Al cathode interface, thus reducing the net dipole. The direct contact of APTES with Al increased the $R_{\mathrm{S}}$ and would have decreased the $J_{\mathrm{sc}}$ however, the $J_{\mathrm{sc}}$ was improved. The $J_{\mathrm{sc}}$ of TFSCs depends on their $R_{\mathrm{s}}$ and $V_{\mathrm{oc}}$. In this study, the addition of the ntype APTES layer improved the $V_{\text {oc }}$ of the TFSCs significantly, which in turn improved the $J_{\mathrm{sc}}{ }^{10,17}$ The polarization effect was not observed when a LiF layer was used at the Al cathode. This prevented the direct contact between APTES and the metal surface. However, it is possible to overall improve the $R_{\mathrm{S}}$ of a device by utilizing different techniques to enhance the front/ rear electrode contact with the underlying silicon layers. Moreover, the presence of a LiF layer in TFSCs reduces the $\varphi$ of the cathodes. ${ }^{27}$ Hence, the LiF/Al cathode devices w/o APTES showed higher $J_{\mathrm{sc}}\left(15.62 \mathrm{~mA} \mathrm{~cm}{ }^{-2}\right)$ and $V_{\mathrm{oc}}(0.78 \mathrm{~V})$ than the $\mathrm{Al}$ cathode devices. The incorporation of APTES in the LiF/Al cathode TFSCs, further enhanced their $V_{\text {oc }}$ to $0.80 \mathrm{~V}$ and reduced the $J_{\mathrm{sc}}$ slightly to $15.51 \mathrm{~mA} \mathrm{~cm}{ }^{-2}$. This discrepancy could be due to two reasons: first that the interfacial dipole was not efficiently generated by APTES, which was not the case as observed by the improvement of $J_{\mathrm{sc}}$ and $V_{\mathrm{oc}}$ in Al cathode device. Also, the creation of an interfacial dipole mainly improves the $V_{\mathrm{bi}}$ as mentioned before ${ }^{28,29}$ and the resulting $V_{\mathrm{oc}}$ for APTES incorporated devices was enhanced. Second, the addition of LiF increased the thickness of the interface between the i-Si layer and the Al cathode, thus increasing the Schottky barrier slightly. This is consistent with the slightly lowered $J_{\mathrm{sc}}$ and increased $V_{\mathrm{oc}}$ of the APTES-treated devices. The low $R_{\mathrm{S}}$ (Table 1) of the APTESbased devices $\left(11.70 \Omega \mathrm{cm}^{2}\right)$ as compared to that of the reference device $\left(12 \Omega \mathrm{cm}^{2}\right)$ confirms that the addition of APTES improved the $V_{\mathrm{bi}}$ of the device by generating an interfacial dipole. The devices with $\left(9.52 \times 10^{3} \Omega \mathrm{cm}^{2}\right)$ and w/o $\left(9.92 \times 10^{3} \Omega \mathrm{cm}^{2}\right)$ APTES showed similar $R_{\mathrm{SH}}$ values, indicating the saturation of $R_{\mathrm{SH}}$. Moreover, the $J-V$ analysis under dark conditions of the devices w/o APTES (with LiF only) showed high leakage currents and hence low stability. This could be attributed to the low thickness and density of the LiF layer w/o APTES, which might have resulted in a poor thin-film coverage. ${ }^{5,9}$ The addition of APTES with LiF reduced the leakage current and improved the $V_{\text {oc }}$ of the devices significantly, which indicates that the addition of APTES improved the LiF layer density by providing adequate thin-film coverage and suppressed the charge recombination. Moreover, in terms of device stability enhancement Wang et $a .^{30}$ presented that the deposition of a thin $(\sim 5 \mathrm{~nm})$ APTES SAM could efficiently inhibit the diffusion of metals into adjacent dielectrics and could simultaneously improve the interfacial adhesion. Also, Ohta K. et al. ${ }^{31}$ demonstrated a combination layer of CoWB and APTES to suppress $\mathrm{Cu}$ diffusion into $\mathrm{SiO}_{2}$. Therefore, we could speculate that the use of APTES/LiF combination in our fabricated a-Si:H TFSC devices could significantly suppress the Al (cathode metal) diffusion into the amorphous silicon layers, thus improving the stability of the devices. We also evaluated the device performance of APTES a-Si:H TFSCS with the reported values of conventional $\mathrm{P}-\mathrm{I}-\mathrm{N}$ device ${ }^{10}$ and another amine-based conjugated polymer interlayer poly[(9,9-bis(3'-( $N, N$-dimethylamino)propyl)-2,7-fluorene)-alt-2,7(9,9-dioctylfluorene)] (PFN)-based solar cell. ${ }^{6}$ In comparison to P-I-N devices, APTES TFSCs demonstrated an enhanced $J_{\mathrm{sc}}$, PCE and $R_{\mathrm{SH}}$ even though FF remained unchanged due to an increased $R_{\mathrm{S}}$ attributed to the different nature of the interfacial contacts. While, in comparison to the PFN devices (amine-based), APTES-treated TFSCs exhibited superior performance in all device parameters. Overall, the APTES-incorporated devices exhibited higher FF (0.61) and PCE $(7.68 \%)$ attributed to their enhanced $V_{\mathrm{bi}}$, electron extraction and stability, thus projecting this toxic-free SAM layer as a competent alternative to other $n$-type/conjugated polymer layers in aSi:H TFSCs.

\section{Conclusions}

In this study, we used APTES as an alternative to n-type layers for a-Si:H TFSCs with improved performance and stability. The facile solution processability of the APTES layer w/o the involvement of any toxic doping gases is highly advantageous for the development of hazard-free solar cells. $\mathrm{The}-\mathrm{NH}_{2}$ groups from the deposited APTES layer reacted with the -OH groups on the i-Si surface through hydrogen bonding to form a positive interfacial dipole towards the i-Si layer (opposite to the direction of electron extraction). The interfacial dipole generated by the APTES layer decreased the Schottky barrier at the interface and 
$\varphi$ of the cathode. This resulted in enhanced electron extraction, suppressed charge recombination, and improved $V_{\mathrm{bi}}$. As a result, the devices with APTES showed enhanced $V_{\text {oc }}(0.80)$, FF (0.61), and PCE (7.68\%) as compared to the devices w/o APTES. The low leakage current of the APTES-based TFSCs presented a strong stability of the devices, which further acknowledges the improvement in device performance and significance of the proposed method.

\section{Author contributions}

S. Y. R. and C. S. K. are corresponding authors designed and conceived this work. H. H. and P. J. J. drafted the manuscript by providing comprehensive analysis. D. H. K., and D. K. C. fabricated the solar cell devices. C. M. L. and J. F. M. participated in the electrical characterizations. A. S. and K. B. C. analyzed the XPS/UPS results in details. M. K. S. provided valuable analysis and discussion.

\section{Conflicts of interest}

The authors declare no conflicts of interests.

\section{Acknowledgements}

This research was supported by Basic Science Research Program through the National Research Foundation of Korea (NRF) funded by the Ministry of Education (NRF-2014R1A6A1030732 and 2017R1A2B4005583). This work was also supported by "Human Resources Program in Energy Technology" of the Korea Institute of Energy Technology Evaluation and Planning (KETEP), granted financial resource from the Ministry of Trade, Industry \& Energy, Republic of Korea (No. 20184030201910). This research was also supported by the "Nano Product Upgrading Program using Electron Beam" through the Gyeongsangnam-do \& Gimhae.

\section{Notes and references}

1 A. Polman, M. Knight, E. C. Garnett, B. Ehrler and W. C. Sinke, Science, 2016, 352, aad4424.

2 H. Kang, G. Kim, J. Kim, S. Kwon, H. Kim and K. Lee, Adv. Mater., 2016, 28, 7821-7861.

3 S. De Wolf, A. Descoeudres, Z. C. Holman and C. Ballif, Green, 2012, 2, 7-24.

4 A. G. Aberle, Thin Solid Films, 2009, 517, 4706-4710.

5 J.-H. Yang, H.-H. Jung, J. Seo, K.-D. Kim, D.-H. Kim, D.-C. Lim, S.-G. Park, J.-W. Kang, M. Song, M.-S. Choi, J.-D. Kwon, K.-S. Nam, Y. Jeong, S.-H. Kwon, P. Y. Chang, Y.-C. Kang, K.-B. Chung, C.-S. Kim, K. S. Lim and S. Y. Ryu, J. Phys. Chem. C, 2013, 117, 23459-23468.

6 S. Y. Ryu, J. hoon Seo, H. Hafeez, M. Song, J. Y. Shin, D. H. Kim, Y. C. Jung and C.-S. Kim, Synth. Met., 2017, 228, 91-98.

7 K. Vats and G. Tiwari, Appl. Energy, 2012, 96, 409-416.

8 J.-H. Yoon, J. Song and S.-J. Lee, Sol. Energy, 2011, 85, 723733.
9 H. H. Jung, J.-D. Kwon, S. Lee, C. Su Kim, K.-S. Nam, Y. Jeong, K.-B. Chung, S. Yoon Ryu, T. Ocak and A. Eray, Appl. Phys. Lett., 2013, 103, 073903.

10 J. Hoon Seo, Y. Chang Park, H. Hwan Jung, H. Woo Lee, S. Yoon Ryu and C. Su Kim, Phys. Chem. Chem. Phys., 2013, 15, 1788-1792.

11 C. Battaglia, X. Yin, M. Zheng, I. D. Sharp, T. Chen, S. McDonnell, A. Azcatl, C. Carraro, B. Ma and R. Maboudian, Nano Lett., 2014, 14, 967-971.

12 J. Bullock, M. Hettick, J. Geissbühler, A. J. Ong, T. Allen, C. M. Sutter-Fella, T. Chen, H. Ota, E. W. Schaler and S. De Wolf, Nat. Energy, 2016, 1, 15031.

13 T. Masuda, N. Sotani, H. Hamada, Y. Matsuki and T. Shimoda, Appl. Phys. Lett., 2012, 100, 253908.

14 W. Wang, E. Schiff and Q. Wang, J. Non-Cryst. Solids, 2008, 354, 2862-2865.

15 M. Song, J.-W. Kang, D.-H. Kim, J.-D. Kwon, S.-G. Park, S. Nam, S. Jo, S. Yoon Ryu and C. Su Kim, Appl. Phys. Lett., 2013, 102, 143303.

16 G. Yang, C. Wang, H. Lei, X. Zheng, P. Qin, L. Xiong, X. Zhao, Y. Yan and G. Fang, J. Mater. Chem. A, 2017, 5, 1658-1666.

17 H. L. Yip, S. K. Hau, N. S. Baek, H. Ma and A. K. Y. Jen, Adv. Mater., 2008, 20, 2376-2382.

18 R. G. Acres, A. V. Ellis, J. Alvino, C. E. Lenahan, D. A. Khodakov, G. F. Metha and G. G. Andersson, J. Phys. Chem. C, 2012, 116, 6289-6297.

19 M. Zhu, M. Z. Lerum and W. Chen, Langmuir, 2011, 28, 416423.

20 T. H. Tran, J.-W. Lee, K. Lee, Y. D. Lee and B.-K. Ju, Sens. Actuators, B, 2008, 129, 67-71.

21 M. Vosgueritchian, M. C. LeMieux, D. Dodge and Z. Bao, ACS Nano, 2010, 4, 6137-6145.

22 Y. Tanizawa and T. Suzuki, J. Chem. Soc., Faraday Trans., 1995, 91, 3499-3503.

23 G. Ingo, N. Zacchetti, D. Della Sala and C. Coluzza, J. Vac. Sci. Technol., A, 1989, 7, 3048-3055.

$24 \mathrm{~J}$. Kim, Investigation of the Formation and Structure of APTES Films on Silicon Substrates, Pike Technologies, 2010, vol. 4, pp. 47-49.

25 MSDS (Materials Safety Data Sheet) for 3Aminopropyltriethoxysilane, https:/pubchem.ncbi.nlm.nih.gov/ compound/3-Aminopropyltriethoxysilane\#section=EcologicalInformation.

26 Z. Starowicz, K. Gawlińska, J. Walter, R. Socha, G. KuleszaMatlak and M. Lipiński, Mater. Res. Bull., 2018, 99, 136-143.

27 C. J. Brabec, S. E. Shaheen, C. Winder, N. S. Sariciftci and P. Denk, Appl. Phys. Lett., 2002, 80, 1288-1290.

28 Z. He, C. Zhong, X. Huang, W. Y. Wong, H. Wu, L. Chen, S. Su and Y. Cao, Adv. Mater., 2011, 23, 4636-4643.

29 B. A. MacLeod, N. E. Horwitz, E. L. Ratcliff, J. L. Jenkins, N. R. Armstrong, A. J. Giordano, P. J. Hotchkiss, S. R. Marder, C. T. Campbell and D. S. Ginger, J. Phys. Chem. Lett., 2012, 3, 1202-1207.

30 F. Wang, Y. Li, Y. Wang and Z. Cao, Nanoscale Res. Lett., 2011, 6, 483.

31 K. Ohta, F. Inoue, T. Shimizu and S. Shingubara, ECS Trans., 2015, 64, 57-61. 